\title{
DESARROLLO, CONSUMO Y ACEPTABILIDAD DE UNA BEBIDA LÁCTEA CON DHA PARA EMBARAZADAS Y NODRIZAS
}

\section{DEVELOPMENT, INTAKE AND ACCEPTABILITY OF A DHA-ENRICHED MILK DRINK FOR PREGNANT AND LACTATING WOMEN}

\author{
Eduardo Atalah S. (1), Gloria Vera A. (1), Gastón Rosselot P. (2), Héctor Araya L. (1,3, 4), \\ Ramón Andreu R. (2), Marcela Alviña W. (3, 4), Marcela Araya B. (5), Vianny Vargas P. (6), \\ Karina Peñafiel W. (7), Catalina Barba G. (8), Tito Pizarro Q. (8) \\ (1) Departamento Nutrición, Facultad Medicina, Universidad Chile. \\ (2) Gerencia Desarrollo \& Calidad, Watt's SA. \\ (3) Departamento Nutrición y Alimentos. Facultad de Farmacia, Universidad de Valparaíso. \\ (4) CREAS. Centro Regional de Estudios en Alimentos Saludables. \\ (5) Alumna Doctorado Nutrición y Alimentos, Universidad de Chile. \\ (6) Nutricionista, Alumna del Programa de Magíster en Salud Pública. \\ (7) Kolkopiw Ltda. \\ (8) Departamento de Alimentos y Nutrición, Ministerio de Salud. Santiago, Chile.
}

\begin{abstract}
DHA is a critical nutrient for pregnant and lactating women, with important functions in brain development and sight acuity of the newborn. In Chile, DHA intake represents less than $30 \%$ of the international recommendation for pregnancy. The objective of this study was to design and evaluate a milk drink for pregnant and lactating women, containing $60 \mathrm{mg}$ of DHA and $11 \mathrm{mg}$ of EPA per $200 \mathrm{ml}$ portion, plus other added vitamins and minerals. A cohort of 177 pregnant women received $2 \mathrm{~kg} / \mathrm{month}$ of the milk drink and compared to a group of 175 women receiving the regular milk distributed by the National Complementary Feeding Program (PNAC), consisting of whole milk powder (26\% fat) fortified with $\mathrm{Fe}, \mathrm{Zn}$, and Vitamin C. Evaluation included acceptability tests, tolerance, and intake of both milks, and measurements were performed at two moments during pregnancy, and after two months of lactation. Both milks had good evaluations in their organoleptic characteristics. Average intake for both groups was $1.8 \pm 1$ cup per day, lower than the three cups per day that were originally defined for the study. During pregnancy, sensory evaluation was better, and intake was higher, than with the regular whole milk, although these differences were not significant from a biological standpoint. During lactation, there were no differences among any of the evaluated parameters. The developed milk drink constitutes a good alternative for improving DHA intake during pregnancy and lactation.
\end{abstract}

Key words: Pregnancy, lactation, DHA, feeding program.

Este trabajo fue recibido el 1 de Septiembre de 2008 y aceptado para ser publicado el 18 de Octubre de 2008

\section{INTRODUCCIÓN}

El embarazo y la lactancia constituyen etapas de alta vulnerabilidad nutricional, con un aumento significativo de las necesidades de casi todos los nutrientes con relación a mujeres en el período preconcepcional $(1,2)$. En Chile ha habido una reducción importante de la prevalencia de bajo peso y aumento de la obesidad en las mujeres, aunque las encuestas alimentarias continúan mostrando bajo consumo de algunos nutrientes críticos, como calcio, hierro, zinc y ácidos grasos omega-3, entre otros (3-6).

De especial importancia es el aporte de ácidos grasos omega-3 de cadena larga. El ácido docosahexaenoico (DHA) juega un rol fundamental en el desarrollo cerebral y de la retina del recién nacido (7-9). El depósito de DHA en la retina y corteza cerebral se produce principalmente 
durante el tercer trimestre de la gestación y en los primeros seis meses de vida extrauterina y un porcentaje importante de los lípidos estructurales tanto del cerebro como de los conos y bastoncitos corresponden a DHA (10-12).

Los niveles de ácidos grasos poliinsaturados de cadena larga (AGPICL) en la madre dependen de su ingesta. Se ha demostrado que la concentración de DHA en las membranas del glóbulo rojo varía ampliamente, en función de los niveles de consumo. Lo mismo ocurre en la leche materna, donde la concentración puede fluctuar entre 0,1 y $1,4 \%$ del total de ácidos grasos, dependiendo del consumo de alimentos de origen marino (13). A nivel mundial se han realizado variadas intervenciones para mejorar los niveles de DHA en el embarazo y lactancia. La mayoría de ellas han sido a través de suplementos y los principales vehículos utilizados son cápsulas, aceites marinos o huevos fortificados con omega-3. La suplementación con DHA se traduce en una reducción de la proporción de recién nacidos de bajo peso al nacer, de partos pretérminos y en una mayor agudeza visual y cuociente intelectual del niño, que se mantiene aún a los 4 años de vida (14-21).

En Chile, a pesar de su extensa costa, el consumo de pescado o de otros alimentos de origen marino es muy bajo, lo que determina un aporte de DHA del orden de $50 \mathrm{mg} /$ día $(22,23)$, muy por debajo de los $200 \mathrm{mg}$ mínimos recomendados para lograr los efectos descritos (12). Ello determina que los niveles de DHA en la leche materna representen menos de la mitad de lo observado a nivel mundial (13). Un estudio previo demostró que una suplementación de la dieta con 160 gramos de un pescado graso en conserva 2 veces por semana (jurel), produjo un aumento significativo de los ácidos grasos omega-3 de la leche materna, que no se mantuvo al suspender la suplementación (24-25). Ello sugiere la necesidad de explorar otras formas de intervención que logren una mejor sustentabilidad.

Un $65 \%$ de las embarazadas y nodrizas del país recibe a través del Ministerio de Salud, una leche en polvo, $26 \%$ de materia grasa, fortificada con hierro, zinc y vitamina $C$, de muy buena aceptabilidad. Sin embargo este producto puede ser mejorado nutricionalmente en función de los nuevos conocimientos en nutrición. Con este objetivo se desarrolló una Bebida Láctea elaborada a partir de leche y cereales hidrolizados, con menor contenido de lípidos y de lactosa en relación a la leche Purita. Además, fortificada con 10 vitaminas, 4 minerales y con adición de ácidos grasos poliinsaturados (AGPI) omega-3 (EPA y DHA).

El objetivo del estudio fue evaluar la aceptabilidad y consumo de la bebida láctea en una cohorte de em- barazadas y nodrizas, información fundamental antes de decidir la eventual incorporación de este alimento al Programa Nacional de Alimentación Complementaria del Ministerio de Salud.

\section{MATERIAL Y MÉTODOS}

Se formuló y elaboró un producto con leche, sólidos lácteos, cereales hidrolizados, aceite vegetal (soya), azúcar, saborizantes, antioxidantes, vitaminas, minerales y AGPI de la familia omega-3. Se evaluó a través de pruebas industriales, la estabilidad de la fuente de AGPI, sobre un soporte de leche descremada o de mezclas de leche y cereales. Después del secado conjunto en una torre spray se analizó el perfil resultante de ácidos grasos y se inició el control de estabilidad durante la vida útil del producto. El producto se envasó con film trilaminado, con atmósfera modificada, desplazándose el oxígeno ambiental por una mezcla de nitrógeno y CO2. A los 60 días se realizaron pruebas sensoriales por un panel de jueces entrenados (parámetros del sabor, texturales y de apariencia), que mostraron un deterioro de la calidad del producto, fundamentalmente por rancidez (sabor y olor extraño).

En función de estos resultados se realizaron nuevas pruebas industriales orientadas a reducir el riesgo de rancidez y prolongar la vida útil del producto. El esfuerzo estuvo orientado a reducir el contenido de hierro y cobre en las materias primas, elementos pro-oxidantes que reaccionan fácilmente con los AGPI y a evaluar el efecto de modificar la fuente y concentración de DHA en el producto. El producto que finalmente se ensayó contiene $400 \mathrm{kcal}, 18 \mathrm{~g}$ de proteínas y $10 \mathrm{~g}$ de grasa por $100 \mathrm{~g}$ producto y aporta $60 \mathrm{mg}$. de DHA y $14 \mathrm{mg}$ de EPA por cada porción de consumo ( $25 \mathrm{~g}$ de producto en polvo disuelto en $200 \mathrm{ml}$ de agua). La fuente de AGPI fue un producto micro encapsulado de la empresa DSM (INFpowerR) que tiene un 7\% de DHA. Incluye además una mezcla de vitaminas y minerales, destacando el aporte de un 25 a 30\% de la dosis diaria de vitamina C, A, ácido fólico y calcio, por porción (tabla 1). El producto no incluye hierro ni cobre para reducir el riesgo de oxidación. Previo a su distribución se sometió a los controles apropiados para evaluar que cumplía con los requisitos microbiológicos, evaluación sensorial y los parámetros tecnológicos de estabilidad, que demuestran que es un alimento apto para el consumo.

El proyecto fue aprobado por el Comité de Ética de la Facultad de Medicina, Universidad de Chile y de los respectivos servicios de salud, donde se desarrolló el estudio. Para el estudio piloto se seleccionaron cinco consultorios (Alberto Bachelet, Apoquindo, Edgardo Enríquez, Norman Voullieme y Sofía Pincheira), perte- 
necientes a tres Servicios de Salud de la Región Metropolitana. En cada uno de ellos se captó una cohorte de embarazadas que cumplieran los siguientes requisitos de ingreso: firma del consentimiento informado por parte de la madre, primer control prenatal antes de las 14 semanas de embarazo, edad mayor de 17 años, embarazo único y ausencia de patologías crónicas que afecten la evolución del embarazo o el crecimiento fetal (enfermedades hepáticas, renales, diabetes, entre otros). Las madres fueron posteriormente seguidas hasta los cuatro meses post parto.

Para definir el tamaño de muestra se estimó 90\% de aceptabilidad de la Leche Purita Fortificada (LPF), una aceptabilidad 10\% menor para la Bebida Láctea (BL), nivel de significación de un 0,05 y poder del test de 80, lo que determinó la necesidad de estudiar 177 mujeres en cada grupo.

La aceptabilidad para los parámetros sabor, olor y color se evaluó utilizando una escala hedónica de 7 puntos, dónde 1 corresponde a "me disgusta extremadamente" y 7 "me gusta extremadamente". Adicionalmente se estimó la aceptabilidad total del producto solicitándole al consumidor que le asignara una nota de 1 a 7 , donde 1 es muy mala y 7 excelente (tabla 2 ). Se consideró como punto de corte 4,5 puntos para definir un parámetro y/o producto como aceptable, de acuerdo a lo utilizado actualmente por el MINSAL para otorgar la adjudicación en la licitación de los productos alimentarios del PNAC y PACAM.

Aleatoriamente las madres que se incorporaron al estudio fueron asignadas a uno de los siguientes grupos: grupo control (GC), que recibió la Leche Purita Fortificada que distribuye actualmente el Programa Nacional de Alimentación Complementaria y grupo intervenido (GI), que recibió la nueva bebida láctea.

En ambos grupos se entregaron dos kilos mensuales del producto, lo que permite el consumo de tres raciones diarias de leche. Se capacitó al personal de salud de

\section{TABLA 1}

\section{Aporte de omega-3, vitaminas y minerales de una porción de la Bebida Láctea como porcentaje de la dosis diaria de referencia para la embarazada}

\begin{tabular}{lccc} 
Nutriente & $\begin{array}{c}\text { Aporte } \\
\mathbf{1 0 0} \mathbf{g}\end{array}$ & $\begin{array}{c}\text { Una porción } \\
\mathbf{2 5} \mathbf{~ g .}\end{array}$ & $\begin{array}{c}\text { Una porción } \\
\text { \% DDR }\end{array}$ \\
\hline EPA (mg) & 54 & 14 & NA* \\
DHA (mg) & 240 & 60 & $30 * *$ \\
Vit A (ug ER) & 480 & 120 & 15 \\
Vit C (mg) & 60 & 15 & 25 \\
Vit. D (ug) & 4 & 1 & 10 \\
Vit E (mg ET) & 30 & 7,5 & 25 \\
Vit. B1 (mg) & 1,4 & 0,35 & 21 \\
Vit. B2 (mg) & 1,6 & 0,40 & 20 \\
Niacina (mg EN) & 16 & 4 & 20 \\
Vit. B6 (mg) & 2 & 0,5 & 20 \\
Ac. Fólico (ug) & 520 & 130 & 16 \\
Vit.B12 (ug) & 5 & 1,3 & 16 \\
& & & 32 \\
Calcio (mg) & 1650 & 413 & 28 \\
Fósforo (mg) & 1480 & 370 & 14 \\
Magnesio (mg) & 250 & 63 & 13 \\
Zinc (mg) & 7,5 & 1,9 & \\
* NA: no aplicable. & & & \\
** No corresponde a la DDR, sino a la recomendación mínima de 200 mg/día propuesta por algunos autores & & \\
(Koletzo B et al. J Perinat Med 2008; $36: 5-14)$. & &
\end{tabular}


cada consultorio (matronas, enfermeras), para realizar las siguientes actividades: selección de las madres para el estudio y obtención del consentimiento informado; mantener el control prenatal según las normas vigentes; dar instrucciones a las madres sobre la preparación de la leche, forma y frecuencia de consumo (tres tazas diarias); encuesta de aceptabilidad, tolerancia y consumo habitual de los productos y los dos días previos a la encuesta, un mes después de ingresar al estudio, en el segundo trimestre del embarazo y a los dos meses post parto en forma habitual; obtener información de la ficha clínica, con relación a edad, paridad, escolaridad, evolución del peso materno y presencia de patologías asociadas.

Los datos fueron procesados con el programa STATA 9.2. En una primera etapa se analizó la normalidad de cada variable a través del test de Shapiro-Wilk. Dado que la mayor parte de las variables tuvieron una distribución normal se utilizaron promedios y desviaciones estándar para las variables continuas y el test de Anova para la comparación entre los grupos. En las variables categóricas se utilizaron distribuciones de frecuencia y el test de chi ${ }^{2}$ para la comparación entre los grupos y en todos los casos se consideraron significativos valores de $\mathrm{p}<0,05$.

\section{RESULTADOS}

Ingresaron al estudio 352 embarazadas (175 GC y 177 GI) a las 10,3 $\pm 2,9$ semanas de gestación, con una edad promedio de 25,6 \pm 7,0 años, escolaridad 10,6 $\pm 2,8$ años y paridad de $1,2 \pm 1,1$. Las características generales y antropométricas de ambos grupos fueron similares y reflejan relativamente bien a las beneficiarias del sistema público de salud.

Durante el seguimiento hubo una disminución de la cohorte inicial, ya sea por cambios de domicilio, inasistencias a control, abortos o por solicitud de las madres de retirarse del estudio. Se logró entrevistar al final del embarazo a 145 mujeres del grupo control (83\% del grupo inicial) y a 104 del grupo intervenido (59\%), siendo la pérdida mayor en el grupo intervenido $(\chi 24,25 \mathrm{p}<0,05)$. No hubo diferencias significativas en las principales variables analizadas en el grupo que

\section{TABLA 2}

\section{Ficha utilizada para evaluar la aceptabilidad de parámetros sensoriales y la aceptabilidad general}

\section{Evaluación de parámetros sensoriales}

Escala

numérica

Descriptor

Parámetros a evaluar

7

6

5

4

3

2

1

Me gusta extremadamente

Me gusta mucho

Me gusta

No me gusta ni me disgusta

Me disgusta

Me disgusta mucho

Me disgusta extremadamente

Evaluación de aceptabilidad general

7

6

5

4

3

2

1
Excelente

Muy bueno

Bueno

Regular

Malo

Muy malo

Inaceptable 
abandonó el estudio respecto al grupo que continuó en la cohorte.

La apreciación de las madres sobre las características organolépticas de ambos productos se presenta en la tabla 3, destacando en general una opinión favorable y muy por encima del punto de corte establecido para definir un producto como aceptable. Sin embargo, durante el embarazo la evaluación fue significativamente mejor en los distintos parámetros, para la Leche Purita Fortificada. A pesar de que las diferencias fueron significativas, dos tercios de las madres del grupo intervenido evalúo el nuevo producto con nota $>6$ y sólo una baja proporción con nota menor de 5. Durante la lactancia la apreciación de ambos productos fue similar.

El consumo promedio diario de leche según la tendencia habitual se presenta en la tabla 4. La mayor parte

\section{TABLA 3}

\section{Puntaje de la evaluación organoléptica de ambos productos en el embarazo y lactancia}

\begin{tabular}{cccc}
\hline Encuesta & $\begin{array}{c}\text { Grupo control } \\
\mathbf{X} \pm \mathbf{D E}\end{array}$ & $\begin{array}{c}\text { Grupo intervenido } \\
\mathbf{X} \pm \mathbf{D E}\end{array}$ & $\mathbf{p}$ \\
Embarazo primera encuesta & & & \\
Sabor & $5,7 \pm 1,7$ & $5,2 \pm 1,7$ & $<0,02$ \\
Olor & $5,7 \pm 1,8$ & $5,1 \pm 1,9$ & $<0,02$ \\
Color & $6,2 \pm 1,5$ & $5,8 \pm 1,5$ & $<0,05$ \\
Nota global & $\mathbf{5 , 9} \pm \mathbf{1 , 4}$ & $\mathbf{5 , 4} \pm \mathbf{1 , 5}$ & $<\mathbf{0 , 0 1}$ \\
Embarazo segunda encuesta & & & $<0,005$ \\
Sabor & $6,0 \pm 1,3$ & $5,6 \pm 1,5$ & $<0,001$ \\
Olor & $6,1 \pm 1,4$ & $5,4 \pm 1,7$ & $<0,001$ \\
Color & $6,5 \pm 1,0$ & $5,8 \pm 1,3$ & $<\mathbf{0 , 0 0 1}$ \\
Nota global & $\mathbf{6 , 2} \pm \mathbf{1 , 1}$ & $\mathbf{5 , 7} \pm \mathbf{1 , 4}$ & \\
Lactancia $(\mathbf{2}$ meses $)$ & & & N.S. \\
Sabor & & $6,0 \pm 1,3$ & N.S. \\
Olor & $6,1 \pm 1,2$ & $6,0 \pm 1,2$ & N.S. \\
Color & $5,8 \pm 1,5$ & $6,3 \pm 1,0$ & N.S. \\
Nota global & $6,3 \pm 1,0$ & $\mathbf{6 , 0} \pm \mathbf{1 , 0}$ &
\end{tabular}

\section{TABLA 4}

Promedio de raciones* diarias de producto según tendencia de consumo durante el embarazo y lactancia

\begin{tabular}{|c|c|c|c|}
\hline Encuesta & $\begin{array}{l}\text { Grupo control } \\
\qquad \mathbf{x} \pm \mathrm{DE}\end{array}$ & $\begin{array}{c}\text { Grupo intervenido } \\
\qquad \mathbf{x} \pm \mathrm{DE}\end{array}$ & $\mathbf{p}$ \\
\hline Embarazo primera encuesta & $1,9 \pm 1,0$ & $1,6 \pm 1,0$ & $<0,05$ \\
\hline Embarazo segunda encuesta & $1,8 \pm 1,1$ & $1,5 \pm 0,9$ & $<0,05$ \\
\hline Lactancia 2 meses & $1,7 \pm 0,9$ & $1,8 \pm 1,0$ & N.S. \\
\hline
\end{tabular}


de las madres consumió entre 1,5 y dos tazas diarias, cifra inferior a las tres tazas programadas de acuerdo a la cantidad de producto que se entregó a cada beneficiaria. El consumo promedio fue mayor para la LPF durante el embarazo ( $\mathrm{p}<0,05)$, lo que no se observó posteriormente en la lactancia.

Los dos días previos al control, no más del $25 \%$ de las madres consumió el equivalente a tres tazas diarias (600 ml o más), con un consumo algo menor en el grupo con Bebida Láctea, que no fue estadísticamente significativo (tabla 5). Destaca un aumento en el consumo de la Bebida Láctea entre el embarazo y la lactancia, reduciéndose en un $60 \%$ las personas que consumían una taza o menos al día $(\mathrm{p}<0,02)$

En ambos grupos se observó una alta frecuencia de meteorismo, constipación, vómitos y cólicos la semana previa a la encuesta, síntomas que fueron disminuyendo hacia el final del embarazo, sin diferencias significativas entre los grupos (tabla 6). La única diferencia observada fue una menor frecuencia de constipación en el grupo intervenido $(\mathrm{p}<0,05)$.

\section{DISCUSIÓN}

El bajo consumo de pescado, principal fuente de AGPI y especialmente de DHA ha sido documentado en diversos estudios nacionales $(3,5,23,24)$. Ello es especialmente preocupante en embarazadas y gestantes, donde el consumo de DHA no supera el 30\% de las recomendaciones mínimas, con todos los eventuales efectos negativos que puede tener en la evolución del embarazo y en el desarrollo cerebral y retiniano del feto.

Diversas estrategias se han utilizado para mejorar el consumo de DHA en la población. Una de ellas es la educación nutricional, orientada a estimular el consumo de pescado. De hecho las guías alimentarias del Ministerio de Salud recomiendan el consumo de pescado como mínimo dos veces por semana, lo que se cumple sólo en el 10\% de la población, según la Encuesta Nacional de Calidad de Vida (6). Casi el 60\% de la muestra estudiada no consumía pescado o lo hacía ocasionalmente, sin grandes diferencias en función de la edad, género o nivel socioeconómico. Para revertir esta situación, no sólo se debe reforzar la educación nutricional, sino que es fundamental además mejorar los sistemas de distribución de los productos marinos y crear incentivos o subsidios para que estos lleguen a bajo precio a la población.

Otra estrategia es el consumo de suplementos de DHA o de aceites marinos, de los cuales existen varios en el mercado. Esta es una buena alternativa, complementaria a la anterior, pero diversas experiencias demuestran

TABLA 5

Distribución según cantidad de leche consumida los dos días previos a la encuesta durante el embarazo y lactancia

\section{Consumo \\ número de tazas/día}

Grupo control

$\%$

27,5

48,1

24,4 $\leq 1,0$

$1,1-2,9$

$\geq 3,0$

\section{Embarazo segunda encuesta}

$$
\begin{gathered}
\leq 1,0 \\
1,1-2,9 \\
\geq 3,0
\end{gathered}
$$

Lactancia 2 meses

$\begin{array}{ccc}\leq 1,0 & 19,2 & 15,6 \\ 1,1-2,9 & 56,4 & 62,2 \\ \geq 3,0 & 24,4 & 22,2\end{array}$


las dificultades para lograr una buena adherencia en este tipo de intervenciones. Un ejemplo claro son los suplementos de hierro en la embarazada, donde persisten tasas relativamente altas de anemia a pesar de que desde hace varias décadas, existen normas de suplementación en forma universal, que se cumplen parcialmente.

La tercera alternativa, es la fortificación de alimentos, estrategia que ha sido muy efectiva en relación a algunos nutrientes: ácido fólico, hierro, vitamina A, fluor, yodo. Es fundamental disponer de un vehículo adecuado, que sea consumido regularmente por la población objetivo y en cantidades que permitan cubrir la brecha que existe entre consumo y necesidades. El Programa Nacional de Alimentación Complementaria (PNAC) ofrece una excelente oportunidad, ya que llega regularmente a dos tercios de las embarazadas y madres en lactancia del país. Recientemente se estudió en el país el efecto de una leche en polvo comercial fortificada con múltiples micronutrientes y ácidos grasos omega-3, demostrando un efecto positivo en el peso de nacimiento (26).

Un desafío tecnológico enorme fue lograr un producto que aporte adecuados niveles de DHA, sea estable y resistente a la oxidación, tenga buenas características organolépticas y cuyo costo no sea muy superior al producto actualmente utilizado por el PNAC. Este proceso demoró más de un año, lográndose finalmente un producto que cumplía con estos requisitos, aunque con una concentración de DHA menor que la inicialmente programada (500 $\mathrm{mg}$ por $100 \mathrm{~g}$ ).

La evaluación organoléptica del nuevo producto fue clasificada como "buena" o "muy buena", aunque en el embarazo tuvo un puntaje inferior al obtenido por la Leche Purita, lo que también se reflejó en un consumo algo menor. Esta menor aceptabilidad podría en parte ser explicada por la dificultad de competir con la Leche Purita Fortificada que tiene excelente imagen, por una insuficiente campaña educativa sobre las ventajas del nuevo producto, por su introducción en las primeras

\section{TABLA 6}

\section{Presencia de síntomas gastrointestinales la semana previa a la encuesta según tipo de producto consumido}

\section{Encuesta}

Embarazada primera encuesta

Cólico

Vómitos

Diarrea

Meteorismo

Constipación

Embarazada segunda encuesta

Cólico

Vómitos

Diarrea

Meteorismo

Constipación

Lactancia 2 meses

Cólico

Vómitos

Diarrea

Meteorismo

Constipación
16,4

24,5

9,4

35,2

23,0

16,0

11,0

6,8

27,7

20,4

25,3
21,8

N.S.

29,3

11,3

N.S.

N.S.

30,1

N.S.

24,8

N.S.

N.S.

N.S.

13,0

N.S.

N.S.

N.S.

$21,4 \quad$ N.S.

N.S.

2,3

N.S.

0,0

N.S.

14,0

N.S:

9,3 
semanas del embarazo cuando aún hay muchos síntomas gastrointestinales o efectivamente por tener características sensoriales menos favorables. Para explorar esta última alternativa se analizó periódicamente el producto por jueces entrenados, obteniéndose buenos resultados. Doce meses después de elaborado y almacenado el producto en los Consultorios, el producto fue trasladado al laboratorio y sometido a otra evaluación sensorial con un panel de jueces entrenados, aplicándose un test pareado de preferencia, que mostró 21 preferencias a favor de la nueva bebida y sólo 3 por la Leche Purita. Es posible que con una buena campaña comunicacional, la aceptación y consumo del producto, aumente en forma significativa.

Preocupa también la mayor tasa de abandono del protocolo en las madres del grupo intervenido, lo que también puede reflejar una menor aceptabilidad. Este hecho en parte puede ser explicado por el consentimiento informado, donde en el grupo intervenido se explicaban las ventajas de los productos marinos en el embarazo, las posibles molestias digestivas que puede ocasionar la leche en algunas personas y se aseguraba a la madre que podía retirarse del estudio en cuanto lo quisiera. Es probable que esta información haya contribuido a retiros injustificados, frente a cualquier síntoma gastrointestinal, que fueron frecuentes en ambos grupos. Otra posible razón puede derivar de las limitaciones de compartir este producto con los hijos u otros miembros de la familia, a diferencia de la leche Purita Fortificada. A favor de las buenas características sensoriales de la bebida láctea está el hecho que aumentó su consumo durante la lactancia, equiparándose a la leche Purita, aunque también podría deberse a que permanecieron en la cohorte sólo aquellas madres que tuvieron mejor opinión del producto.

Otro hecho que preocupa es el bajo nivel de consumo de leche observado en ambos grupos, que representa menos de la mitad de las 4-5 raciones diarias recomendadas por el Ministerio de Salud. Sin embargo en la interpretación de estos datos, debe tenerse en consideración que no se incluyeron en la encuesta otros lácteos (yogur, queso, quesillo), que pueden aportar un tercio o más del consumo diario de este grupo de alimentos. En la etapa pregestacional el consumo fue aún más bajo, mejorando en forma significativa con la incorporación al programa, lo que demuestra la importancia de los programas alimentarios para mejorar la calidad de la alimentación en la población de menores recursos.

\section{RESUMEN}

El DHA es un nutriente crítico en mujeres embarazadas y en lactancia, con importantes beneficios en el desarrollo cerebral y agudeza visual del lactante. En
Chile el consumo de DHA representa menos del $30 \%$ de las recomendaciones internacionales para el embarazo. El objetivo del estudio fue diseñar y evaluar una bebida láctea para embarazadas y nodrizas adicionada de $60 \mathrm{mg}$ de DHA y 11 de EPA por porción de consumo (200 ml), además de estar fortificada con vitaminas y minerales. Se estudió una cohorte de 177 embarazadas que recibieron $2 \mathrm{Kg}$. mensuales de bebida láctea en comparación con un grupo control de 175 embarazadas que recibió el producto actualmente en uso en el Programa Nacional de Alimentación Complementaria (leche $26 \%$ de materia grasa, fortificada con $\mathrm{Fe}, \mathrm{Zn}$ y vit $\mathrm{C}$ ). Se evaluó aceptabilidad, tolerancia y consumo de ambos productos en dos oportunidades en el embarazo y a los 2 meses posparto. Ambos alimentos fueron bien evaluados en sus características organolépticas. El consumo promedio de ambos grupos fue 1,8 $\pm 1,0$ tazas diarias, inferior a las 3 tazas programadas. Durante el embarazo la evaluación sensorial y el consumo fueron mayores para la leche entera, aunque las diferencias no fueron importantes desde el punto de vista biológico. Durante la lactancia no hubo diferencias en ninguno de los parámetros evaluados. Se concluye que la bebida láctea es una buena alternativa para mejorar el consumo de DHA durante el embarazo y lactancia.

Términos claves: embarazo, lactancia, omega-3, DHA, programa alimentario.

Dirigir la correspondencia a:

Dr.

Eduardo Atalah S.

Departamento de Nutrición

Facultad de Medicina

Universidad de Chile

Independencia 1027, Santiago, Chile

Fono (562) 9786239

Fax (562) 7355581

Correo electrónico: eatalah@med.uchile.cl

Agradecimientos: Al financiamiento del Proyecto FONIS SA04I2089.

\section{BIBLIOGRAFÍA}

1. Uauy R, Atalah E, Barrera C, Behnke E. Alimentación y nutrición durante el embarazo. En: Burrows, Castillo C, Atalah E, Uauy R eds. Guías de alimentación de la mujer. Imprenta La Nación, Santiago 2001, 53-74

2. Atalah E, Gómez C, Salazar G, Castillo C. Alimentación y nutrición durante la lactancia y período intergestacional. En: Burrows, Castillo C, Atalah E, Uauy R eds. Guías de alimentación de la mujer. 
Imprenta La Nación, Santiago 2001, 75-90.

3. Díaz C, Valeria V, Oscar H y Biolley H, Emma E. Ingesta dietaria de nutrientes críticos en embarazadas. Rev Chil Nutr 2005; 32: 225-231

4. Atalah, E, Castro R. Obesidad materna y riesgo reproductivo. Rev Méd Chile 2004; 132: 923-930.

5. Durán E, Soto D, Labrana AM, Pradenas F. Adecuación dietética de micronutrientes en embarazadas. Rev Chil Nutr 2007; 34: 321-329.

6. Ministerio de Salud. Encuesta de Calidad de Vida 2006. http://epi.minsal.cl/epi/html/sdesalud/calidaddevida2006/index.htm, consultado julio 2008

7. Innis SM, Friesen RW. Essential n-3 fatty acids in pregnant women and early visual acuity maturation in term infants. Am J Clin Nutr 2008 ; 87:548-57.

8. Katzen-Luchenta J. The declaration of nutrition, health, and intelligence for the child-to-be. Nutr Health 2007; 19: 85-102.

9. Hadders-Algra M. Prenatal long-chain polyunsaturated fatty acid status: the importance of a balanced intake of docosahexaenoic acid and arachidonic acid. J Perinat Med. 2008; 36:101-9.

10. Valenzuela A, Nieto S. Acido docosahexaenoico (DHA) en el desarrollo fetal y en la nutrición materno-infantil. Rev Méd Chile 2001;129:1203-1211.

11. Hadders-Algra M, Bouwstra H, van Goor SA, Dijck-Brouwer DA, Muskiet FA. Prenatal and early postnatal fatty acid status and neurodevelopmental outcome. J Perinat Med 2007;35 Suppl 1:S28-34

12. Koletzko B, Lien E, Agostoni C, Böhles H, Campoy C, Cetin I, Decsi T, Dudenhausen JW, Dupont C, Forsyth S, Hoesli I, Holzgreve W, Lapillonne A, Putet G, Secher NJ, Symonds M, Szajewska H, Willatts P, Uauy R; World Association of Perinatal Medicine Dietary Guidelines Working Group. The roles of long-chain polyunsaturated fatty acids in pregnancy, lactation and infancy: review of current knowledge and consensus recommendations. J Perinat Med 2008; 36:5-14.

13. Brenna JT, Varamini B, Jensen RG, Diersen-Schade DA, Boettcher JA, Arterburn LM. Docosahexaenoic and arachidonic acid concentrations in human breast milk worldwide. Am J Clin Nutr 2007; 85: 1457-64.

14. Innis SM. Human milk: maternal dietary lipids and infant development. Proc Nutr Soc 2007; 66: 397-404.

15. Sanjurjo P, Ruiz-Sanz JI, Jimeno P, AldamizEchevarria L, Aquino L, Matorras R, Esteban J, Banque M. Supplementation with docosahexaenoic acid in the last trimester of pregnancy: maternalfetal biochemical findings. J Perinat Med 2004; 32
(2):132-6.

16. Bergmann RL, Haschke-Becher E, Klassen-Wigger P, Bergmann KE, Richter R, Dudenhausen JW, Grathwohl D, Haschke F. Supplementation with 200 $\mathrm{mg}$ /day docosahexaenoic acid from mid-pregnancy through lactation improves the docosahexaenoic acid status of mothers with a habitually low fish intake and of their infants. Ann Nutr Metab 2008; 52:157-66.

17. Bakker EC, Hornstra G, Blanco CE, Vles JS. Relationship between long-chain polyunsaturated fatty acids at birth and motor function at 7 years of age. Eur J Clin Nutr. http://www.nature.com/ejen/journal/vaop/ncurrent/abs/1602971a.html, Consultado Octubre 2008.

18. Muthayya S, Dwarkanath P, Thomas T, Ramprakash S, Mehra R, Mhaskar A, Mhaskar Thomas A, Bhat S, Vaz M, Kurpad AV. The effect of fish and omega-3 LCPUFA intake on low birth weight in Indian pregnant women. Eur J Clin Nutr http://www.nature. com/ejcn/journal/vaop/ncurrent/abs/1602933a.html, Consultado Octubre 2008.

19. Dunstan JA, Mitoulas LR, Dixon G, Doherty DA, Hartmann PE, Simmer K, Prescott SL. The effects of fish oil supplementation in pregnancy on breast milk fatty acid composition over the course of lactation: a randomized controlled trial. Pediatr Res 2007; 62: 689-94.

20. Krauss-Etschmann S, Shadid R, Campoy C, Hoster E, Demmelmair H, Jiménez M,Gil A, Rivero M, Veszprémi B, Decsi T, Koletzko BV; Nutrition and Health Lifestyle (NUHEAL) Study Group. Effects of fish-oil and folate supplementation of pregnant women on maternal and fetal plasma concentrations of docosahexaenoic acid and eicosapentaenoic acid: a European randomized multicenter trial. Am J Clin Nutr 2007; 85:1392-400.

21. Parra-Cabrera S, Moreno-Macias H, MendezRamirez I, Schnaas L, Romieu I. Maternal dietary omega fatty acid intake and auditory brainstemevoked potentials in Mexican infants born at term: cluster analysis. Early Hum Dev 2008; 84:51-7

22. Atalah E, Araya H, Rosselot G, Pinheiro AC, Araya M, Vargas V. Consumo de pescado, mariscos, EPA y DHA en gestantes de la Región Metropolitana. Primer Congreso Chileno de Salud Pública, Determinantes de la Salud, Resumen 17, Santiago, 2007.

23. Aguirre C. Evaluación del consumo de ácidos grasos de cadena larga en la dieta de adultos chilenos. Primer Congreso Chileno de Salud Pública, Determinantes de la Salud, Resumen 32, Santiago, 
2007.

24. Gaete M, Atalah E, Araya J. Efecto de la suplementación de la dieta de la madre durante la lactancia con ácidos grasos omega 3 en la composición de los lípidos de la leche. Rev Chil Pediatr 2002; 73 : 239-247.

25. Gaete M, Atalah E. Niveles de LC-PUFA n-3 en la leche materna después de incentivar el consumo de alimentos marinos. Rev Chil Pediatr 2003; 74: 158-165.

26. Mardones F, Urrutia MT, Villarroel L, Rioseco A, Castillo O, Rozowski J, Tapia JL y cols. Effects of a dairy product fortified with multiple micronutrients and omega-3 fatty acids on birth weight and gestation duration in pregnant Chilean women. Public Health Nutr 2008; 11: 30-40 